\title{
Elementary presentation of self-consistent intermediate Hamiltonians and proposal of two totally dressed singles and doubles configuration interaction methods
}

\author{
J. P. Malrieu \\ Laboratoire de Physique Quantique, URA 505 du CNRS, Université Paul Sabatier, 118, route de \\ Narbonne, 31062 Toulouse Cedex, France

\begin{abstract}
I. Nebot-Gil and J. Sanchez-Marin
Departamento de Quimica Fisica, Facultad de Ciencias Quimicas, c/Dr Moliner 50, 46100 Burjasot (Valencia), Spain
\end{abstract}

(Received 13 July 1993; accepted 1 October 1993)

\begin{abstract}
Intermediate Hamiltonians are effective Hamiltonians which are defined on an $\mathrm{N}$-dimensional model space but which only provide $n<N$ exact eigenvalues and the projections of the corresponding eigenvectors onto the model space. For a single root research, the intermediate Hamiltonian may be obtained from the restriction of the Hamiltonian to the model space by an appropriate, uniquely defined dressing of the diagonal energies or of the first column. Approximate self-consistent dressings may be proposed. The simplest perturbative form gives the same result as the original 2 nd order intermediate Hamiltonian or the "shifted $B_{k}$ " technique but it is of easier implementation. Self-consistent inclusion of higher order exclusion principle violating corrections greatly improves the results, especially for nearly degenerate problems, as shown on several illustrative applications. Possible generalizations to enlarged or reduced model spaces are discussed.
\end{abstract}

\section{INTRODUCTION}

The basic structure of the electronic many-body problem has been formulated through the RayleighSchrödinger (RS) perturbative expansion starting from the independent-particle approximation, and has led to the fundamental linked cluster theorem. ${ }^{1}$ These results make clear the origin of the size-inconsistency of truncated variational configuration interaction $(\mathrm{CI})$ calculations, and the physical likeliness of coupled cluster expansions ${ }^{2}$ of the wave function

$$
\psi=\exp S \phi_{0},
$$

where $S$ keeps a simple form [linear combination of single and double excitations for instance (CC-SD)]. Both RS perturbative expansion and the $\mathrm{CC}$ one are based on a single reference, at this stage. In molecular physics, the Hartree-Fock (HF) $\phi_{0}$ determinant becomes degenerate with some doubly (and eventually more) excited determinant when chemical bonds are broken, and these near degeneracies induce divergences of the perturbation series and question the validity of the CC expansion. It is in principle possible to move to quasidegenerate perturbation theory (QDPT) and a generalized linked cluster theorem has actually been established by Brandow ${ }^{3}$ for a very special type of model space. The model space must be complete, i.e., accept all possible distributions of a fixed number of active electrons $n_{a}$ into a fixed set of active monoelectronic functions $N a$, thus generating $C_{N a}^{n_{a}}$ determinants, some of which are very exotic and high in energy. The chemists call this space a valence complete active space (CAS) which is unvariant under unitary transformations of valence spin orbitals and complete for the symmetry species considered. They have immediately noticed that QDPT had no chance to converge, in general, due to the occurrence of near degeneracies between some determinants of the outer space (acting as "intruder states") and some determinants of the model space. ${ }^{4}$ These near degeneracies are unavoidable in practice, and condemn the use of a size-consistent QDPT for the computation of reliable potential energy surfaces (PES). On the other hand multireference coupled cluster theories have been the subject of an intense collective effort, ${ }^{5}$ but the solutions are very difficult and their implementation is not straightforward.

In this context, a new tool has been proposed some years ago by one of us (J.P.M.) and co-workers, namely, the intermediate effective Hamiltonians. ${ }^{6}$ The $\mathrm{N}$-dimensional model space is partitioned into a main model space, of dimension $n_{a}$, and an intermediate model space, of dimension $n_{i}=N-n_{a}$, and the intermediate Hamiltonian $\widetilde{H}$, built onto the whole model space, only delivers $n_{m}$ exact eigenvectors onto the whole model space. A perturbative expansion (generalized degenerate perturbation theory) was proposed at this time, and other variants have been suggested by different authors, ${ }^{7}$ since, as explained below, the intermediate Hamiltonians are not uniquely defined.

The strength of intermediate Hamiltonians is their ability to accept near-degeneracies between the intermediate model space and the outer space, thus solving the intruder state problem. ${ }^{8}$

The present paper proposes in Sec. II a very simple presentation of intermediate Hamiltonians dedicated to the research of a single root. It shows that they are obtained by changing (or dressing) the elements of the truncated Hamiltonians matrix $P H P$, where $P$ is the projector on the 
model space. We briefly discuss the flexibility of the dressing, and propose an elementary formulation of the dressing in terms of the coefficients of the eigenstate on the outer space determinants. This trivial expression is in principle academic, but will receive a large series of applications, beyond the present work. In Sec. III, we concentrate on a fourth-order variation/perturbation combination, which, for CI problems, consists in an iterative dressing of the SDCI matrix by the effect of the triples and quadruples. This dressing may be of perturbative type or inspired by coupled cluster expansions, but the presented work is limited to the perturbative aspect.

The simplest version gives the same solution than the so-called shifted $B_{k}$ approximation ${ }^{9}$ (which is a 2 nd order intermediate Hamiltonian, with a full dressing of the CI matrix) but is less memory consuming. A sophisticated version takes into account high-order EPV corrections in a self-consistent manner and this addition makes possible to treat accurately single bond breaking, despite the monodeterminantal character of the main model space, as shown through a few test calculations (Sec. IV). Possible generalizations to enlarged or reduced model spaces are discussed in Sec. V.

\section{THE SELF-CONSISTENT INTERMEDIATE HAMILTONIANS}

\section{A. Generalities}

Let us suppose that one has defined an $n$-dimensional model space $S$ of projector $P$

$$
P=\sum_{\substack{i=0 \\ i \in S}}^{n-1}\left|\phi_{i}\right\rangle\left\langle\phi_{i}\right|,
$$

the functions $\phi_{i}$ being orthonormal single determinants or configurations. Also assume that one only searches one eigenvalues $\epsilon_{0}$ and the corresponding eigenvector $\psi_{0}$

$$
H \psi_{0}=\epsilon_{0} \psi_{0}
$$

or more precisely its projection into the model space, $P \psi_{0}$. This means that one desires to build an intermediate Hamiltonian (according to a previous definition ${ }^{6}$ ) built on $S$,

$$
\bar{H}=P \bar{H} P,
$$

such that

$$
\begin{aligned}
& \bar{H} \bar{\psi}_{0}=\epsilon_{0} \bar{\psi}_{0}, \\
& \bar{\psi}_{0}=P \psi_{0} .
\end{aligned}
$$

Of course this condition fixes one energy and $(n-1)$ coefficients, and does not determine $\bar{H}$ uniquely, while the effective Hamiltonians are entirely defined by their spectral definition, for instance, for Bloch's Hamiltonians, ${ }^{10}$

$$
H^{\mathrm{eff}}=\sum_{k=1, n}\left|P \psi_{k}\right\rangle \epsilon_{k}\left\langle P \psi_{k}^{1}\right|
$$

One has a great flexibility in the definition of $\bar{H}$. The partitioning technique ${ }^{11}$ proposes one choice

$$
\bar{H}=P H P+P H Q\left(\epsilon_{0}-Q H Q\right)^{-1} Q H P,
$$

which presents the difficulty of the inversion of $Q H Q, Q$ being the projector on the outer space $(Q=1-P)$, and which modifies all the matrix elements of the truncated matrix

$$
\left\langle\phi_{i}|\bar{H}| \phi_{j}\right\rangle \neq\left\langle\phi_{i}|H| \phi_{j}\right\rangle \quad \forall i, j .
$$

Now we shall assume that one of the basis functions, $\phi_{0}$, is a good approximation to $\psi_{0}$. By that we do not assume that $\left\langle\psi_{0} \mid \phi_{0}\right\rangle$ is large, which is not compulsory (even for the convergence of a nondegenerate perturbation from $\left.\phi_{0}\right)$, but simply that $\psi_{0}$ is the eigenvector having the largest component on $\phi_{0}$,

$$
\left|\left\langle\psi_{0} \mid \phi_{0}\right\rangle\right|>\left|\left\langle\psi_{i} \mid \phi_{0}\right\rangle\right| \quad \forall i \neq 0
$$

and reciprocally

$$
\left.\left|\left\langle\psi_{0} \mid \phi_{0}\right\rangle\right|\right\rangle\left|\left\langle\psi_{0} \mid \phi_{i}\right\rangle\right| \quad \forall i \neq 0,
$$

which is a very weak condition. Then, $\phi_{0}$ will define our main model space, of projector $P_{m}$,

$$
P_{m}=\left|\phi_{0}\right\rangle\left\langle\phi_{0}\right|
$$

and the other determinants of our model space define the intermediate model space, of projector $P_{i}$,

$$
P_{i}=P-P_{m}=\sum_{\substack{k \neq 0 \\ \in S}}\left|\phi_{k}\right\rangle\left\langle\phi_{k}\right| .
$$

It is evident that if one knows the exact eigenergy $E_{0}$ and the components of the exact eigenvector on the model space, i.e., in the intermediate normalization

$$
P \psi_{0}=\phi_{0}+\sum_{i \in S} C_{i} \phi_{i},
$$

it is always possible to define a diagonal operator $\Delta$ defined on $S$, which we shall call the diagonal dressing operator,

$$
\left\langle\phi_{k}|\Delta| \phi_{l}\right\rangle=0 \text { if } k \neq l,
$$

such that

$$
P(H+\Delta) P \psi_{0}=\epsilon_{0} \psi_{0}
$$

This eigenequation becomes, for the line $i$,

$$
\begin{gathered}
\left\langle\phi_{i}|H| \phi_{0}\right\rangle+\sum_{\substack{j \in S \\
\neq i, 0}} C_{j}\left\langle\phi_{i}|H| \phi_{j}\right\rangle+C_{i}\left(\left\langle\phi_{i}|H| \phi_{i}\right\rangle\right. \\
\left.+\left\langle\phi_{i}|\Delta| \phi_{i}\right\rangle-\epsilon_{0}\right)=0
\end{gathered}
$$

and uniquely defines

$$
\left\langle\phi_{i}|\Delta| \phi_{i}\right\rangle=\epsilon_{0}-\left\langle\phi_{i}|H| \phi_{i}\right\rangle-\sum_{j \neq i}\left\langle\phi_{i}|H| \phi_{j}\right\rangle \frac{C_{j}}{C_{i}}
$$

(if $C_{i} \neq 0$; if $C_{i}=0,\left\langle\phi_{i}|\Delta| \phi_{i}\right\rangle$ may be kept to zero). This is an academic view, although it may be used to define "dressed energies" for instance to analyze in a pictorial manner the effect of the nondynamical correlation as a change of the effective energies of the valence compo- 
nents. ${ }^{12}$ In general we do not know the exact eigenvector, which we are trying to approach, and the preceding remark is simply a proof of unique existence of the diagonal dressing operator, analogous to the spectral definition of $H^{\text {eff }}$ in the quasidegenerate perturbation theory.

An alternative solution would consist in the definition of a "column" dressing, modifying the matrix elements between $\phi_{0}$ and the $\phi_{i}$ 's, i.e., of the first column of the Hamiltonian matrix

$$
\left\langle\phi_{k}\left|\Delta^{\prime}\right| \phi_{l}\right\rangle=\left\langle\phi_{k}\left|\Delta^{\prime}\right| \phi_{0}\right\rangle \delta_{j} .
$$

Referring to the eigenequations for $H$ and $H+\Delta^{\prime}$ one obtains

$$
\begin{aligned}
\left\langle\phi_{i}\left|\Delta^{\prime}\right| \phi_{0}\right\rangle= & -\left[\left\langle\phi_{i}|H| \phi_{0}\right\rangle+\sum_{j \in S \neq i, 0} C_{j}\left\langle\phi_{i}|H| \phi_{j}\right\rangle\right. \\
& \left.+C_{i}\left(\left\langle\phi_{i}|H| \phi_{i}\right\rangle-\epsilon_{0}\right)\right] .
\end{aligned}
$$

This approach has two drawbacks (1) it is non-Hermitian; (2) it will introduce matrix elements between $\phi_{0}$ and all $\phi_{i}$ 's even if they differ by a large number of spin-orbitals.

Other dressings have been evoked in a more specialized context. $^{13}$

\section{B. The basic equation}

Actually the exact eigenequation, $H \psi_{0}=\epsilon_{0} \psi_{0}$, is written, for line $i$,

$$
\begin{aligned}
& \left\langle\phi_{i}|H| \phi_{0}\right\rangle+\sum_{\substack{j \neq i, 0 \\
\epsilon S}} C_{j}\left\langle\phi_{i}|H| \phi_{j}\right\rangle+C_{i}\left(\left\langle\phi_{i}|H| \phi_{i}\right\rangle-\epsilon_{0}\right) \\
& \quad+\sum_{\alpha \notin S} C_{\alpha}\left\langle\phi_{i}|H| \phi_{\alpha}\right\rangle=0 .
\end{aligned}
$$

Comparison of Eq. (16) with Eq. (13) immediately gives another definition of the dressing matrix element

$$
\left\langle\phi_{i}|\Delta| \phi_{i}\right\rangle=\left(C_{i}\right)^{-1} \sum_{\alpha \notin S} C_{\alpha}\left\langle\phi_{i}|H| \phi_{\alpha}\right\rangle .
$$

This equation still looks academic since one does not know the coefficients $C_{\alpha}$ outside of the model space. But there are cases where one may have good guesses of the coefficients $C_{\alpha}$, relative to the determinants $\phi_{\alpha}$ interacting with at least one determinant of the model space. In general the coefficients $C_{\alpha}$ are functions of the coefficients $C_{k}$, plus additional information. Then, if one knows a function $f$ such that

$$
\left\{C_{\alpha}\right\}=f\left\{C_{k}\right\},
$$

the dressing $\Delta$ may formally be written

$$
\{\Delta\}=g\left\{C_{k}\right\}
$$

i.e., the dressing is a function of the coefficients and the intermediate Hamiltonian $\bar{H}$ becomes a self-consistent Hamiltonian satisfying the matrix equation

$$
\widetilde{H}(C)=P[H+\Delta(C)] P C=\epsilon_{0} C .
$$

The problem is then nonlinear.
An altcrnative formulation consists in introducing a vector $\Delta^{\prime}(C)$ the $i$ th component of which is

$$
\Delta_{i}^{\prime}=\left\langle\phi_{i}|\Delta| \phi_{i}\right\rangle C_{i}=\sum_{\alpha \notin S} C_{\alpha}\left\langle\phi_{i}|H| \phi_{\alpha}\right\rangle
$$

and the problem becomes a vectorial equation

$$
\left(P H P-\epsilon_{0}\right) \mathbf{C}+\Delta^{\prime}(C)=0 .
$$

Notice that this procedure may be considered as a nonHermitian dressing of $P H P$ by addition of extra terms on the first column matrix elements $\left\langle\phi_{i}|H| \phi_{0}\right\rangle$,

$$
\left\langle\phi_{i}|\bar{\Delta}| \phi_{0}\right\rangle=\sum_{\alpha} C_{\alpha}\left\langle\phi_{i}|H| \phi_{\alpha}\right\rangle,
$$

and solution of the resulting self-consistent equation

$$
\left\{P[H+\bar{\Delta}(C)] P-\epsilon_{0}\right\} C=0 .
$$

This formulation has the advantage that the dressing $\left\langle\phi_{i}|\bar{\Delta}| \phi_{0}\right\rangle$ is always possible while $\left\langle\phi_{i}|\Delta| \phi_{i}\right\rangle$ cannot be defined if $C_{i}=0$ (or exceedingly small), at least at the first iteration of the process (for instance, in the configuration interaction problem if $\phi_{0}$ is the ground state determinant and $\phi_{i}$ is a triply or quadruply excited determinant in the configuration interaction problem).

Also remark that the method is not restricted to the ground state, and it may be applied to an excited state as well, changing the reference function $\phi_{0}$. But it is restricted to a state-by-state dressing, with the intermediate Hamiltonians being different for the different roots of the same model space.

\section{PERTURBATIVE FORMULATION}

\section{A. The simplest scheme}

Let us assume that the model space $S$ involves, besides $\phi_{0}$, all the determinants interacting with $\phi_{0}$ through $H$,

$$
P \phi_{i}=\phi_{i} \quad \text { if }\left\langle\phi_{i}|H| \phi_{0}\right\rangle \neq 0 \text {. }
$$

This means that the components of the first-order wavefunction belong to $S$. Then if one considers the secondorder perturbed wave-function $\psi^{(2)}$, using a yet unspecified zeroth-order Hamiltonian,

$$
H_{0}=\sum_{i \in S} E_{i}^{0}\left|\phi_{i}\right\rangle\left\langle\phi_{i}\left|+\sum_{\alpha \notin S} E_{\alpha}^{0}\right| \phi_{\alpha}\right\rangle\left\langle\phi_{\alpha}\right|
$$

its components in the outer space are given by

$$
\begin{aligned}
Q \psi^{(2)} & =\frac{Q_{0}}{E_{0}^{0}-H_{0}} V \psi^{(1)} \\
& =\sum_{\alpha \notin S}\left(E_{0}^{0}-E_{\alpha}^{0}\right)^{-1} \sum_{k \in S}\left\langle\phi_{\alpha}|H| \phi_{k}\right\rangle C_{k}^{(1)}
\end{aligned}
$$

so that one may write, to a better approximation, replacing $C_{k}^{(1)}$ by $C_{k}$,

$$
C_{\alpha}=\left(\sum_{k \in S} C_{k}\left\langle\phi_{\alpha}|H| \phi_{k}\right\rangle\right)\left(E_{0}^{0}-E_{\alpha}^{0}\right)^{-1}
$$

and 


$$
\left\langle\phi_{i}|\Delta| \phi_{i}\right\rangle=C_{i}^{-1} \sum_{\alpha \notin S} \frac{\left\langle\phi_{i}|H| \phi_{\alpha}\right\rangle}{E_{0}^{0}-E_{\alpha}^{0}} \sum_{k \in S}\left\langle\phi_{\alpha}|H| \phi_{k}\right\rangle C_{k} .
$$

In practice one sees that the evaluation of the coefficients $C_{\alpha}$, and therefore of the dressing of the diagonal energies, combines variation and perturbation (for the last step, going from the model space to the outer space). Thus it is an iterative process since the coefficients depend on the dressing and the dressing depends on the coefficients.

As briefly mentioned before, the division by the coefficients $C_{i}$, some of which may be very small, may be considered as a source of troubles despite the fact that (i) $C_{i}^{-1}$ multiplies small quantities (namely a part of the third order energy); (ii) the division by $C_{i}$ may be avoided since one proceeds later to a multiplication by $C_{i}$ [see Eq. (20) or (22)]. And actually it may happen that the ground state physically relevant root is not the lowest eigenvalue of the dressed matrix.

The first column dressing

$$
\left\langle\phi_{i}|\bar{\Delta}| \phi_{0}\right\rangle=\sum_{\alpha \notin S} \frac{\left\langle\phi_{i}|H| \phi_{\alpha}\right\rangle}{E_{0}^{0}-E_{\alpha}^{0}} \sum_{k \in S}\left\langle\phi_{\alpha}|H| \phi_{k}\right\rangle C_{k}
$$

avoids these difficulties but is non-Hermitian.

\section{B. Comparison with previous formulations}

At this stage, the method should be related to the application of the original version of intermediate Hamiltonians to this particular definition of the main and intermediate model spaces. In that version the second-order dressing concerned all the matrix elements between functions belonging to the model space and was given by

$$
\begin{aligned}
\left\langle\phi_{i}\left|\Delta^{\prime \prime}\right| \phi_{j}\right\rangle= & \sum_{\alpha \notin S} \frac{\left\langle\phi_{i}|H| \phi_{\alpha}\right\rangle\left\langle\phi_{\alpha}|H| \phi_{j}\right\rangle}{E_{0}^{0}-E_{\alpha}^{0}} \\
& \forall \phi_{i}, \quad \phi_{j} \in S,
\end{aligned}
$$

$E_{0}^{0}$ being the zeroth order energy for the functions of the main model space. Of course $P(H+\Delta) P \neq P\left(H+\Delta^{\prime \prime}\right) P$ but one may verify that the two dressed matrices have a common root and eigenvector since the eigenequation for lines $i$ are identical. For $\Delta^{\prime \prime}$ it gives

$$
\begin{gathered}
\left\langle\phi_{i}|H| \phi_{0}\right\rangle+\sum_{j \neq i}\left(\left\langle\phi_{i}|H| \phi_{j}\right\rangle\right. \\
\left.+\sum_{\alpha \notin S} \frac{\left\langle\phi_{i}|H| \phi_{\alpha}\right\rangle\left\langle\phi_{\alpha}|H| \phi_{j}\right\rangle}{E_{0}^{0}-E_{\alpha}^{0}}\right) C_{j}+\left(\left\langle\phi_{i}|H| \phi_{i}\right\rangle\right. \\
\left.\quad+\sum_{\alpha \notin S} \frac{\left\langle\phi_{i}|H| \phi_{\alpha}\right\rangle\left\langle\phi_{\alpha}|H| \phi_{i}\right\rangle-\epsilon_{0}}{E_{0}^{0}-E_{\alpha}^{0}}\right) C_{i}=0
\end{gathered}
$$

while for $\Delta$ it is written

$$
\begin{aligned}
& \left\langle\phi_{i}|H| \phi_{0}\right\rangle+\sum_{j \neq i}\left\langle\phi_{i}|H| \phi_{j}\right\rangle C_{j}+\left[\left\langle\phi_{i}|H| \phi_{i}\right\rangle+C_{i}^{-1}\right. \\
& \left.\quad \times \sum_{\alpha \notin S} \frac{\left\langle\phi_{i}|H| \phi_{\alpha}\right\rangle}{E_{0}^{0}-E_{\alpha}^{0}}\left(\sum_{j}\left\langle\phi_{\alpha}|H| \phi_{j}\right\rangle C_{j}\right)-\epsilon_{0}\right] C_{i}=0 .
\end{aligned}
$$

Therefore, at this stage the present self-consistent diagonal dressing is identical to our previously proposed nondiagonal noniterative dressing, when the intermediate model space involves all the components interacting with the main function $\phi_{0}$. Such a formulation had been proposed a long time ago by Davidson as a "shifted- $B_{k}$ approximation," by reference to a previous work by Shavitt et $a l^{14}$ derived from the partitioning technique. The interest of our present self-consistent diagonal version is twofold.

(i) It does not require a significant memory in the computation. In the original version one had to store all $\left\langle\phi_{i}\left|\Delta^{\prime}\right| \phi_{j}\right\rangle$ elements, and while the undressed matrix was sparce, the dressed matrix was full. For instance one can consider the electronic correlation problems, $\phi_{0}$ being the HF approximation. The intermediate model space is spanned by all the doubly excited configuration $\phi\left(\begin{array}{c}a b \\ r s\end{array}\right)$ where $r$ and $s$ are occupied MOs, $a$ and $b$ are virtual MOs. Then most pairs of doubly excited determinants $\phi\left({ }_{r s}^{a b}\right)$ and $\phi\left(\begin{array}{c}c d \\ z u\end{array}\right)$ do not interact, $\left\langle\phi_{i}|H| \phi_{j}\right\rangle=0$, while there is always at least a quadruply excited determinant $\phi\left({ }_{r s t u}^{a b c d}\right)$ which interacts with both determinants and contribute to $\left\langle\phi_{i}\left|\Delta^{\prime \prime}\right| \phi_{j}\right\rangle$.

(ii) It is much more rapid, as noticed and exploited by Maynau and Heully, ${ }^{15}$ to perform an outer loop on the triples and quadruples $\phi_{\alpha}$ and to determine their coefficient $C_{\alpha}$ than to generate the appropriate $\phi_{\alpha}$ 's for all couples $\phi_{i}$, $\phi_{j}$ (i.e., the $\phi_{\alpha}$ 's interacting with both $\phi_{i}$ and $\phi_{j}$ ) as done in the diagrammatic version of the CIPSI algorithm. ${ }^{16}$ This question will be addressed more in detail in a forthcoming work.

The cost to pay is the iterative character of the dressing, but the convergence is so rapid that the present formulation is much more convenient.

The nondiagonal noniterative 2 nd order intermediate Hamiltonian built on the SDCI model space has been demonstrated to be qualitatively size-extensive, i.e., to give a correlation energy proportional to the number of particles. ${ }^{17}$ So that we may assert that the here-proposed iterative diagonal dressing insures the size-extensivity. However, at this stage the method is not strictly separable, i.e., does not give exactly the energy of an $A B$ supersystem where $A$ and $B$ do not interact as the sum of the energies of $A$ and $B$.

The method should also be compared to the selfconsistent size-consistent singles and double CI, recently proposed by one of us (J.P.M.) and co-workers. ${ }^{13,18}$ In this method, which may be seen as an improved coupled electron pair approximation ${ }^{19}$ (CEPA), the dressing of the excited determinants is given by 


$$
\left\langle\phi_{i}|\Delta| \phi_{i}\right\rangle=\sum_{\substack{j \\ D_{j}^{+} \phi_{i} \neq 0}} C_{j}\left\langle\phi_{0}|H| \phi_{j}\right\rangle,
$$

where $D_{j}^{+}$is a (single or) double excitation and $\phi_{j}$ $=D_{j}^{+} \phi_{0}$. This simple dressing insures the size-consistence and the strict separability of the energy of a supersystem $A \ldots B$ into the sum of the energies of $A$ and $B$ when the MOs localize on systems $A$ or $B$. It is worthwhile comparing Eqs. (17) and (29). Equation (29) is obtained from Eq. (17) by simply (a) reducing $\phi_{\alpha}$ to the quadruples when dressing the doubles. $\phi_{t}$ actually interacts with all the quadruples $\phi_{\alpha}$ which are obtained from $\phi_{i}$ by another (disjoint) double excitation. If $\phi_{\alpha}$ is a quadruple interacting with $\phi_{i}\left(\left\langle\phi_{\alpha}|H| \phi_{i}\right\rangle \neq 0\right)$ then one may find a double excitation $D_{j}^{+}$such that

$$
D_{j}^{+} \phi_{i}=\phi_{\alpha}
$$

and

$$
\left\langle\phi_{\alpha}|H| \phi_{i}\right\rangle=\left\langle D_{j}^{+} \phi_{0}|H| \phi_{0}\right\rangle .
$$

(b) Reducing then $C_{\alpha}$ to a single term when dressing $\phi_{i}$,

$$
C_{\alpha}=C_{i} C_{j} \text { if } \phi_{\alpha}=D_{j}^{+} D_{i}^{+} \phi_{0}
$$

instead of its complete expansion (see below Sec. V).

The (SC) ${ }^{2}$ SDCI algorithm dresses each excited determinant by the unlinked effects of the outer space only, while we here propose a full dressing involving both linked and unlinked contributions from the triples and quadruples.

\section{Improved version: Addition of higher orders EPV corrections}

A slightly better evaluation of the coefficient $C_{\alpha}$ (and therefore a better dressing) may be obtained as follows. One might have added $\phi_{\alpha}$ to the intermediate model space, and the eigenequation would have been written for the line $\phi_{\alpha}$,

$$
\begin{gathered}
\sum_{k \in S} C_{k}\left\langle\phi_{\alpha}|H| \phi_{k}\right\rangle+\left(\left\langle\phi_{\alpha}|H| \phi_{\alpha}\right\rangle-E\right. \\
\left.+\left\langle\phi_{\alpha}|\Delta| \phi_{\alpha}\right\rangle\right) C_{\alpha}=0 .
\end{gathered}
$$

Of course one does not know $\left\langle\phi_{\alpha}|\Delta| \phi_{\alpha}\right\rangle$. But one may approximate it by considering the above-mentioned selfconsistent size-consistent CIs. ${ }^{18}$ In this method and for that CI, the dressing would be [cf. Eq. (29)],

$$
\left\langle\phi_{\alpha}|\Delta| \phi_{\alpha}\right\rangle=E-\left\langle\phi_{0}|H| \phi_{0}\right\rangle+\mathrm{EPV}_{\alpha} \text {, }
$$

where $E$ is the exact energy and $\mathrm{EPV}_{\alpha}$ represent the exclusion principle violating corrections

$$
\begin{aligned}
& \mathrm{EPV}_{\alpha}=-\sum_{i} C_{i}\left\langle\phi_{0}|H| \phi_{i}\right\rangle, \\
& D_{i}^{+} \phi_{\alpha}=0 .
\end{aligned}
$$

Equation (31) is obtained by noticing that in Eq. (29) the right-hand member is equal to the correlation energy plus the $\mathrm{EPV}_{i}$ contribution for $\phi_{i}$.
This last term sums the effect of all the single and double excitations which cannot act on $\phi_{\alpha}$, due to the Pauli principle. Then it comes out from Eqs. (30) and (31) that

$$
\begin{aligned}
C_{\alpha}= & \sum_{k \in S} C_{k}\left\langle\phi_{\alpha}|H| \phi_{k}\right\rangle /\left[\left\langle\phi_{0}|H| \phi_{0}\right\rangle\right. \\
& \left.-\left(\left\langle\phi_{\alpha}|H| \phi_{\alpha}\right\rangle+\mathrm{EPV}_{\alpha}\right)\right] .
\end{aligned}
$$

The derivation naturally induces the choice of the EpsteinNesbet definition of the zeroth-order Hamiltonian

$$
H_{0} \phi_{I}=\left\langle\phi_{I}|H| \phi_{I}\right\rangle \phi_{I} \quad \forall I=0, k, \alpha
$$

but we now have added higher order effects through the EPV corrections. The practical implementation of the EPV corrections through the introduction of one-, two-, and three-dimensional arrays takes benefit of a previous work on the infinite summation of EPV diagrams ${ }^{20}$ and has been explicited when presenting the (SC) ${ }^{2} \mathrm{CI}$ algorithms. ${ }^{18}$

Finally,

$$
\begin{aligned}
& \left\langle\phi_{i}|\Delta| \phi_{i}\right\rangle \\
& =C_{i}^{-1} \sum_{\alpha \nsubseteq S} \frac{\left\langle\phi_{i}|H| \phi_{\alpha}\right\rangle}{\left\langle\phi_{0}|H| \phi_{0}\right\rangle-\left(\left\langle\phi_{\alpha}|H| \phi_{\alpha}\right\rangle+\mathrm{EPV}_{\alpha}\right)} \\
& \quad \times \sum_{k \in S} C_{k}\left\langle\phi_{\alpha}|H| \phi_{k}\right\rangle
\end{aligned}
$$

or

$$
\left\langle\phi_{i}|\bar{\Delta}| \phi_{0}\right\rangle=\left\langle\phi_{i}|\Delta| \phi_{i}\right\rangle C_{i} .
$$

The practical advantage of this formulation will be illustrated below, but it is clear that the EPV corrections slow the possible divergence when $\phi_{\alpha}$ and $\phi_{0}$ tend to become too close in energy. If one of the doubles $\phi_{k}$ becomes degenerate with $\phi_{0}$ some triples and quadruples $\phi_{\alpha}$ involving the double excitation $D_{k}^{+}$become low in energy, but the effect of this double excitation $D_{k}^{+}$is impossible on these $\phi_{\alpha}$ 's so that the energy denominator increases. Even if two doubly excited determinants $D_{k}^{+} \phi_{0}$ and $D_{l}^{+} \phi_{0}$ tend to become degenerate with $\phi_{0}$, the quadruply excited determinant $\phi_{\alpha}=D_{k}^{+} D_{l}^{+} \phi_{0}$, which also tend to become degenerate with $\phi_{0}$, will not lead to a zero-energy denominator, due to the large $\mathrm{EPV}_{\alpha}$ corrections.

Notice that here, if the EPV terms are calculated from the variational coefficients [cf. Eq. (32)] the method is really self-consistent, and it might not be transformed, as occurred for the simplest version, into a noniterative fullmatrix dressing.

One may easily demonstrate the following statements.

Statement 1: If $Q$ is the projector on the outer space $(1=P+Q)$, and if $Q H Q$ is diagonal in the basis of the $\phi_{\alpha}$ 's, then the self-consistent diagonal dressing including the EPV terms gives the exact energy.

The proof goes through the following remark.

(i) if $Q H Q$ is diagonal it means that $\left\langle\phi_{\alpha}|H| D_{j}^{+} \phi_{\alpha}\right\rangle$ $=0, \forall j$ and therefore that $D_{j}^{+} \phi_{\alpha}=0$. Hence $\mathrm{EPV}_{\alpha}$ $=-\Sigma_{j} C_{j}\left\langle\phi_{0}|H| \phi_{j}\right\rangle=-x$, where $x$ is the searched correlation energy. Then, 


$$
\left\langle\phi_{i}|\Delta| \phi_{i}\right\rangle=C_{i}^{-1} \sum_{\alpha \notin S} \sum_{j \in S} \frac{\left\langle\phi_{i}|H| \phi_{\alpha}\right\rangle\left\langle\phi_{\alpha}|H| \phi_{j}\right\rangle C_{j}}{E-E_{\alpha}^{0}} .
$$

(ii) This is equivalent, according to a preceding remark to a nondiagonal dressing

$$
\begin{aligned}
\left\langle\phi_{i}\left|\Delta^{\prime}\right| \phi_{i}\right\rangle & =\sum_{\alpha \in S} \frac{\left\langle\phi_{i}|H| \phi_{\alpha}\right\rangle\left\langle\phi_{\alpha}|H| \phi_{i}\right\rangle}{E-E_{\alpha}^{0}}, \\
\left\langle\phi_{i}\left|\Delta^{\prime}\right| \phi_{j}\right\rangle & =\sum_{\alpha \in S} \frac{\left\langle\phi_{i}|H| \phi_{\alpha}\right\rangle\left\langle\phi_{\alpha}|H| \phi_{j}\right\rangle}{E-E_{\alpha}^{0}},
\end{aligned}
$$

which is exactly the partitioning technique dressing [Eq. (6)] for the case where $Q H Q$ is diagonal in the basis of the $\phi_{\alpha}$ 's. The partitioning technique equation is exact, therefore $E$ is exact. This is a rather special case. It implies that the problem concerns four electrons only, otherwise there is no reason that all $\left\langle\phi_{\alpha}|H| D_{j}^{+} \phi_{\alpha}\right\rangle$ are zero. But we shall mention an amazing application of that statement in the next section.

Statement 2: For the problem of $n \mathrm{H}_{2}$ molecules in a minimal basis set, the self-consistent dressing including the EPV terms gives the exact energy.

The undressed matrix may be written in a new basis

$$
n^{-1 / 2} \sum_{i} \phi_{i}\left(\begin{array}{cc}
0 & \sqrt{n} h \\
\sqrt{n} h & \Delta E
\end{array}\right),
$$

where $h$ is the interaction between the HF determinant and any doubly excited determinant, if all molecules are identical. The simplest perturbation will give a dressed matrix

$$
\left(\begin{array}{cc}
0 & \sqrt{n} h \\
\sqrt{n} h & \Delta E-(n-1) \frac{h^{2}}{\Delta E}
\end{array}\right)
$$

whose eigenvalue behaves as $-(n-1)\left(h^{2} / \Delta E\right)$, which means that the energy per molecule becomes $-h^{2} / \Delta E$. In that case the variational step becomes asymptotically useless, and the correlation energy evaluation behaves as purely perturbative. On the contrary if one introduces the EPV correction, the equation becomes

$$
\left(\begin{array}{cc}
0 & \sqrt{n} h \\
\sqrt{n} h & \Delta E-(n-1) \frac{h^{2}}{\Delta E-C h}
\end{array}\right)
$$

where $C$ is the coefficient on each doubly excited determinant, and the eigenvalue is $n C h$, as desired.

Statement 3: However this dressing does not give the exact separability for independent electron pairs $(1, \ldots, i, \ldots, n)$ when nonminimal basis sets are used. It only gives the sum of the self-consistent 2 nd order energies, as calculated according to Ref. 24 , for the separate subsystems.

$$
E(1, \ldots, i, \ldots, n)=\sum_{i=1, n} \epsilon_{(\mathrm{SC})^{2}}^{2}(i)
$$

We let the reader demonstrate this result.
All these statements lead to the conclusion that the presently proposed total dressing of the SDCI matrix insures the correct behavior of the energy with the number of particles, although the strict separability is not insured.

\section{ILLUSTRATIVE EXAMPLES}

We shall only give a few numcrical examples, with special attention to difficult situations where a degeneracy tends to occur between $\phi_{0}$ and one of the doubly excited configurations. One might believe that since our method is basically a single-reference scheme, it will be unable to manage that (near) degeneracy. We want to illustrate the ability of our totally-dressed SDCI algorithm to treat correctly such physical situations.

\section{A. The $\mathrm{H}_{4}$ model problem: The rectangle into square deformation}

This model problem has been the critical test-ground for a series of methods, especially for coupled cluster algorithms. ${ }^{21}$ If a rectangular $\mathrm{H}_{4}$ conformation is distorted towards the square $(R=r)$,

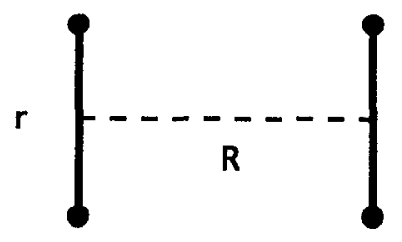

a degeneracy occurs between the $b_{1}$ and $b_{2}$ MOs

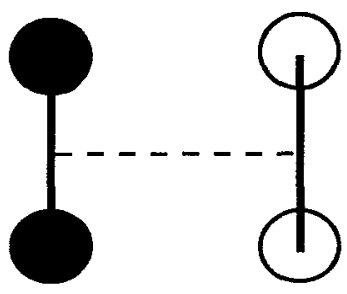

b 1

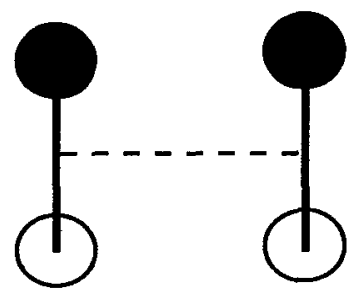

b 2 and the determinant $\phi_{0}=\left|a_{1}^{2} b_{1}^{2}\right|$ becomes degenerate with a doubly excited determinant $\phi_{i}=\left|a_{1}^{2} b_{2}^{2}\right|$. This is a case where in the minimal basis set the singles and triples are not of the relevant symmetry so that the outer space reduces to a unique quadruply excited configuration $\left|b_{2}^{2} a_{2}^{2}\right|$. Then as it has been demonstrated above, $Q H Q$ being diagonal, the total-dressing with EPV gives the exact solution. This will no longer be true for nonminimal basis sets and we have performed a similar calculation in a double zeta basis set.

The results appear in Table $\mathrm{I}$ and Fig. 1. The $r$ value was kept at 1.40158 a.u. Starting from large $R$ values where the SDCI error is small but non-negligible, the error increases when $R$ decreases. The self-consistent sizeconsistent SDCI scheme, which only adds the unlinked effects of the triples and quadruples reduces the error by a factor 2 at most. The incorporation of the linked effects in our perturbative total dressing (TD1) reduces the error by one order of magnitude at least, even for $R \leqslant r$, and the incorporation of the EPV in the dressing (TD1EPV) re- 
TABLE I. Rectangular $\mathrm{H}_{4}$ system in a double zeta basis set as a function of $R$ (a.u.) for $r=1.40158$ a.u. FCI energies (in a.u.), and errors to FCI.

\begin{tabular}{lcccccc}
\hline \multicolumn{1}{c}{$R$} & FCI & FCI-SCF & FCI-SDCI & FCI-(SC) $)^{2}$ CI & FCI-TD1 & FCI-TD1EPV \\
\hline 1.2 & -1.793989 & -0.058914 & -0.001472 & -0.000984 & 0.000011 & 0.000016 \\
1.24 & -1.802647 & -0.061854 & -0.001891 & -0.001266 & 0.000022 & 0.000016 \\
1.28 & -1.810103 & -0.065750 & -0.002597 & -0.001744 & 0.000043 & 0.000018 \\
1.32 & -1.817366 & -0.071340 & -0.003868 & -0.002607 & 0.000101 & 0.000008 \\
1.36 & -1.826048 & -0.079990 & -0.006193 & -0.004168 & 0.000230 & 0.000020 \\
1.4 & -1.83821 & -0.093570 & -0.009926 & -0.006563 & 0.000487 & 0.000083 \\
1.40158 & -1.838784 & -0.094227 & -0.010096 & -0.006668 & 0.000499 & $8.6 \mathrm{E}-05$ \\
1.42 & -1.846002 & -0.08808 & -0.008235 & -0.005493 & 0.000364 & $4.8 \mathrm{E}-05$ \\
1.44 & -1.854827 & -0.08282 & -0.006576 & -0.004415 & 0.000248 & $1.5 \mathrm{E}-05$ \\
1.46 & -1.86444 & -0.078775 & -0.005302 & -0.003571 & 0.000166 & $-7 \mathrm{E}-06$ \\
1.48 & -1.874579 & -0.075666 & -0.004356 & -0.002938 & 0.00011 & $-2.3 \mathrm{E}-05$ \\
1.5 & -1.885016 & -0.073248 & -0.003658 & -0.002468 & $7.2 \mathrm{E}-05$ & $-3.2 \mathrm{E}-05$ \\
1.52 & -1.89558 & -0.071337 & -0.003141 & -0.00212 & $4.6 \mathrm{E}-05$ & $-3.8 \mathrm{E}-05$ \\
1.54 & -1.906151 & -0.069797 & -0.002793 & -0.001859 & $2.8 \mathrm{E}-05$ & $-4.2 \mathrm{E}-05$ \\
1.56 & -1.916645 & -0.068533 & -0.002457 & -0.001659 & $1.5 \mathrm{E}-05$ & $-4.4 \mathrm{E}-05$ \\
1.58 & -1.927007 & -0.067476 & -0.002227 & -0.001505 & $6 \mathrm{E}-06$ & $-4.5 \mathrm{E}-05$ \\
1.6 & -1.937201 & -0.066578 & -0.002046 & -0.001419 & $-1 \mathrm{E}-06$ & $-4.6 \mathrm{E}-05$ \\
1.8 & -2.027364 & -0.061597 & -0.001303 & -0.00088 & $-2.7 \mathrm{E}-05$ & - SE-05 \\
2 & -2.096821 & -0.059056 & -0.001088 & -0.00073 & $-3.4 \mathrm{E}-05$ & $-5.2 \mathrm{E}-05$ \\
2.2 & -2.149662 & -0.057225 & -0.000979 & -0.000652 & $-3.8 \mathrm{E}-05$ & $-5.2 \mathrm{E}-05$ \\
2.4 & -2.18966 & -0.055767 & -0.000911 & -0.000603 & $-3.9 \mathrm{E}-05$ & $-5.2 \mathrm{E}-05$ \\
2.6 & -2.219768 & -0.054576 & -0.000864 & -0.000569 & $-3.8 \mathrm{E}-05$ & $-5.1 \mathrm{E}-05$ \\
2.8 & -2.242283 & -0.053601 & -0.000828 & -0.000544 & $-3.7 \mathrm{E}-05$ & $-4.9 \mathrm{E}-05$ \\
20. & -2.303002 & -0.049850 & -0.000701 & -0.000446 & $-4.7 \mathrm{E}-05$ & $-5.6 \mathrm{E}-05$ \\
\hline \hline
\end{tabular}

duces the error by another order of magnitude for $R<1.1 r$ (the errors are negligible for both TD1 and TD1 EPV for $R>1.1 r$ ). The ability of our procedure to go through the avoided crossing $R=r$ is nicely demonstrated.

Table I also reports the energies for two $\mathrm{H}_{2}$ noninteracting molecules. The error to full $\mathrm{CI}$ is the deviation to additivity since for each subsystems SDCI gives the exact energy. One sees that in this case, due to the use of delocalized MOs, the (SC) ${ }^{2}$ SDCI procedure deviates from the strict additivity (by about half of the error of SDCI). Let us remember that $(\mathrm{SC})^{2}$ SDCI is strictly additive when localized MOs are used, but is not unvariant under unitary transformation of MOs. The deviation to additivity is reduced by one order of magnitude when the total dressing by triples and quadruples is performed. In order to check whether this behavior remained true for more correlated systems we have repeated the calculation for $R=20$ u.a., $r=2.0$ u.a. The total correlation energy is then 0.068552 a.u., and the errors of SDCI and (SC) ${ }^{2}$ SDCI are 0.002147 and 0.001499 a.u., respectively, and the total dressing again reduces the error by a factor $10(0.000143 \mathrm{a.u}$. for TD1, 0.000206 a.u. for TD1+EPV $)$. These errors $(\sim 0.1$ $\mathrm{kcal} \mathrm{mol}^{-1}$ ) are below what is considered to be the chemical accuracy.

\section{B. Breaking of a single bond in a four-electron problem}

As a test example we have studied the $\mathrm{T}$-shaped $\mathrm{Li}_{4}$ problem, fixing an atom on the top of $\mathrm{Li}_{3}$ isosceles triangle and studying the dependance of the energy on the length $R$ of the axial $\mathrm{Li} \cdots \mathrm{Li}$ bond. The geometry may be characterized as follows $x_{1}=3$ a.u., $y_{1}=0, x_{2}=3$ a.u., $y_{2}$

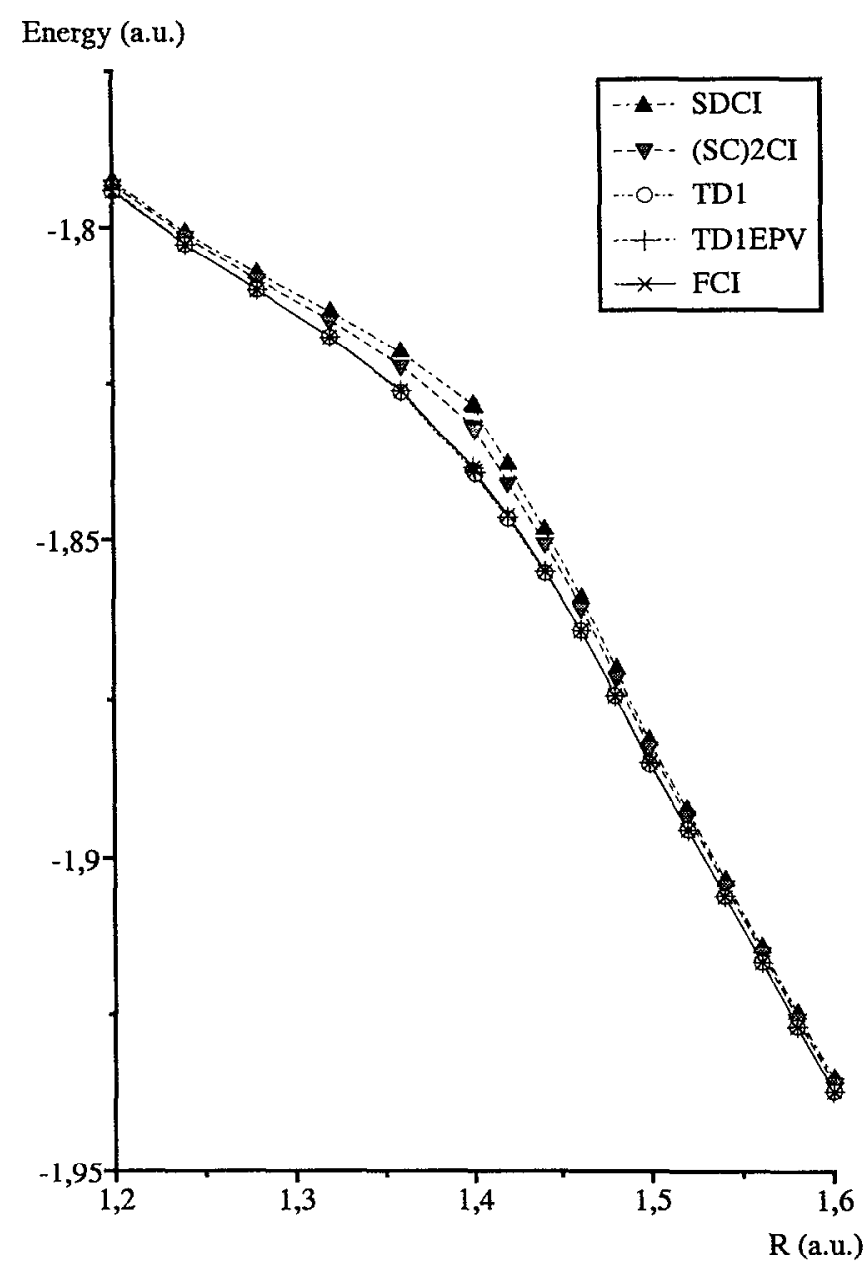

FIG. 1. Evolution of the energy in the rectangular $\mathrm{H}_{4}$ problem in a double zeta basis set, as a function of $R$ for $r=1.40158$ a.u. 
TABLE II. Characteristics of the T-shaped $\mathrm{Li}_{4}$ potential curve (fixed $\mathrm{Li}_{3}$ isoceles triangle, lengthening of the axial $\mathrm{Li}-\mathrm{Li}$ bond) in a double zeta basis set. All quantities are in $\mathrm{cm}^{-1}$, except $R_{e}$ (a.u.).

\begin{tabular}{lcccccc}
\hline \hline & SCF & SDCI & $(\text { SC })^{2}$ SDCI & TD1 & TD1-EPV & FCI \\
\hline$R_{e}$ & 5.359 & 5.544 & 5.576 & 5.620 & 5.605 & 5.611 \\
$\omega_{e}$ & 257 & 206 & 193 & 184 & 187 & 186 \\
$\omega_{e} x_{e}$ & 0.878 & 2.034 & 2.290 & 2.968 & 2.401 & 2.576 \\
$\alpha_{e}$ & 0.00068 & 0.00110 & 0.00131 & 0.00156 & 0.00137 & 0.00140 \\
$D_{e}$ & 22598 & 4643 & 3069 & 1554 & 2732 & 2649 \\
\hline \hline
\end{tabular}

$=0, x_{3}=0, y_{3}=7$ a.u., $x_{4}=0, y_{4}=7$ a.u. This may be seen as a model for a single bond in the presence of other electron pair $(s)$. The calculations have been performed with various basis sets $(1 s)(1 s, 1 p)$, and $(2 s)$ which give similar behaviors so that we only report those relative to the last basis set. The results appear in Tables II and III and in Fig. 2. Comparison is made between SDCI, the size consistent self-consistent SDCI, the perturbative total dressing without and with EPV, and the full CI results.

The first element of comparison concerns the potential well region, which may be analyzed by its "spectroscopic constants" if the $\mathrm{Li}_{3}$ triangle is considered as a pseudoatom (cf. Table II). It is clear that going from SDCI to (SC) ${ }^{2}$ SDCI, then to our total dressings without and with EPV corrections systematically improves all the characteristics, the equilibrium distance the curvature $\left(\omega_{e}\right)$ and the anharmonicity. The effect of the EPV corrections is not negligible. Regarding the total energies one sees from Table III that at short interatomic distances the (SC) ${ }^{2}$ SDCI energies were not significantly better than those of SDCI. The total dressing brings the energy very close to the exact one ( error $\mid<10^{-4}$ a.u. for 4 bohr $<R<6.2$ bohr), but below it. The EPV correction pushes the energy above the exact one, with a somewhat larger but constant error of $\sim 2.10^{-4}$ a.u.
When the bond is broken (large $R$ values) the SDCI energies become much too high, with an asymptotic error of $1.1 \times 10^{-2}$ a.u. The (SC) ${ }^{2}$ SDCI method repairs the major part of that defect since the asymptotic error falls down to $3.8 \times 10^{-3}$ a.u. The proposed total dressing here does not diverge, despite its perturbative character, but it gives energies that are too low, with an asymptotic error of $4.1 \times 10^{-3}$ a.u. The inclusion of the EPV correction is sufficient to give an accurate behavior in the asymptotic region since the error remains $0.7 \times 10^{-3}$ a.u. The dissociation energies $D_{e}$ reported in Table II reflect these differences in the asymptotic behaviors and show the ability of our total dressing with EPV to treat the bond breaking to a high accuracy despite (i) its single reference nature and (ii) its perturbative character.

\section{DISCUSSION}

The present work has proposed a very simple presentation of self-consistent intermediate Hamiltonians for the research of a single root. This root is not necessarily the lowest one, but it is the eigenvector which has the largest component on the determinant chosen as main model space or reference. Since the choice of that determinant is

TABLE III. T-shaped $\mathrm{Li}_{4}$ problem with an increase of the axial bond length $R$ (in a.u.). FCI energy and errors to FCI (in a.u.).

\begin{tabular}{lllllll}
\hline \hline$R$ & FCI & FCI-SCF & FCI-SDCI & FCI-(SC) ${ }^{2} \mathrm{CI}$ & FCI-TD1 & FCI-TD1EPV \\
\hline 5 & -0.80014 & -0.02699 & -0.00183 & -0.00142 & $7 \mathrm{E}-06$ & -0.000142 \\
5.2 & -0.802142 & -0.027808 & -0.001988 & -0.001524 & $1.5 \mathrm{E}-05$ & -0.000155 \\
5.4 & -0.803485 & -0.028671 & -0.002156 & -0.001628 & $2.6 \mathrm{E}-05$ & -0.000169 \\
5.6 & -0.804296 & -0.02958 & -0.002332 & -0.001729 & $4 \mathrm{E}-05$ & -0.000184 \\
5.8 & -0.804687 & -0.030539 & -0.002519 & -0.00183 & $5.5 \mathrm{E}-05$ & -0.0002 \\
6 & -0.804745 & -0.031547 & -0.002716 & -0.001928 & $7.5 \mathrm{E}-05$ & -0.000217 \\
6.2 & -0.804548 & -0.032607 & -0.002923 & -0.002024 & $9.8 \mathrm{E}-05$ & -0.000234 \\
6.4 & -0.804158 & -0.033721 & -0.003141 & -0.002119 & 0.000125 & -0.000253 \\
6.6 & -0.803627 & -0.034889 & -0.003369 & -0.002211 & 0.000156 & -0.000273 \\
6.8 & -0.802999 & -0.036111 & -0.003607 & -0.002302 & 0.000193 & -0.000294 \\
7 & -0.802307 & -0.037386 & -0.003853 & -0.00239 & 0.000237 & -0.000315 \\
7.2 & -0.801582 & -0.038714 & -0.004108 & -0.002477 & 0.000287 & -0.000337 \\
7.4 & -0.800846 & -0.040092 & -0.00437 & -0.002562 & 0.000344 & -0.00036 \\
7.6 & -0.800117 & -0.041518 & -0.004638 & -0.002646 & 0.000408 & -0.000383 \\
7.8 & -0.799409 & -0.042988 & -0.004911 & -0.002727 & 0.000481 & -0.000407 \\
8 & -0.798731 & -0.044497 & -0.005187 & -0.002807 & 0.000562 & -0.000431 \\
9 & -0.79598 & -0.052452 & -0.006552 & -0.00316 & 0.00109 & -0.000551 \\
10 & -0.794315 & -0.06057 & -0.007769 & -0.003426 & 0.001814 & -0.000652 \\
20 & -0.79269 & -0.101112 & -0.011178 & -0.003793 & 0.003431 & -0.00073 \\
40 & -0.79269 & -0.113498 & -0.011511 & -0.003784 & 0.004146 & -0.000627 \\
\hline \hline
\end{tabular}




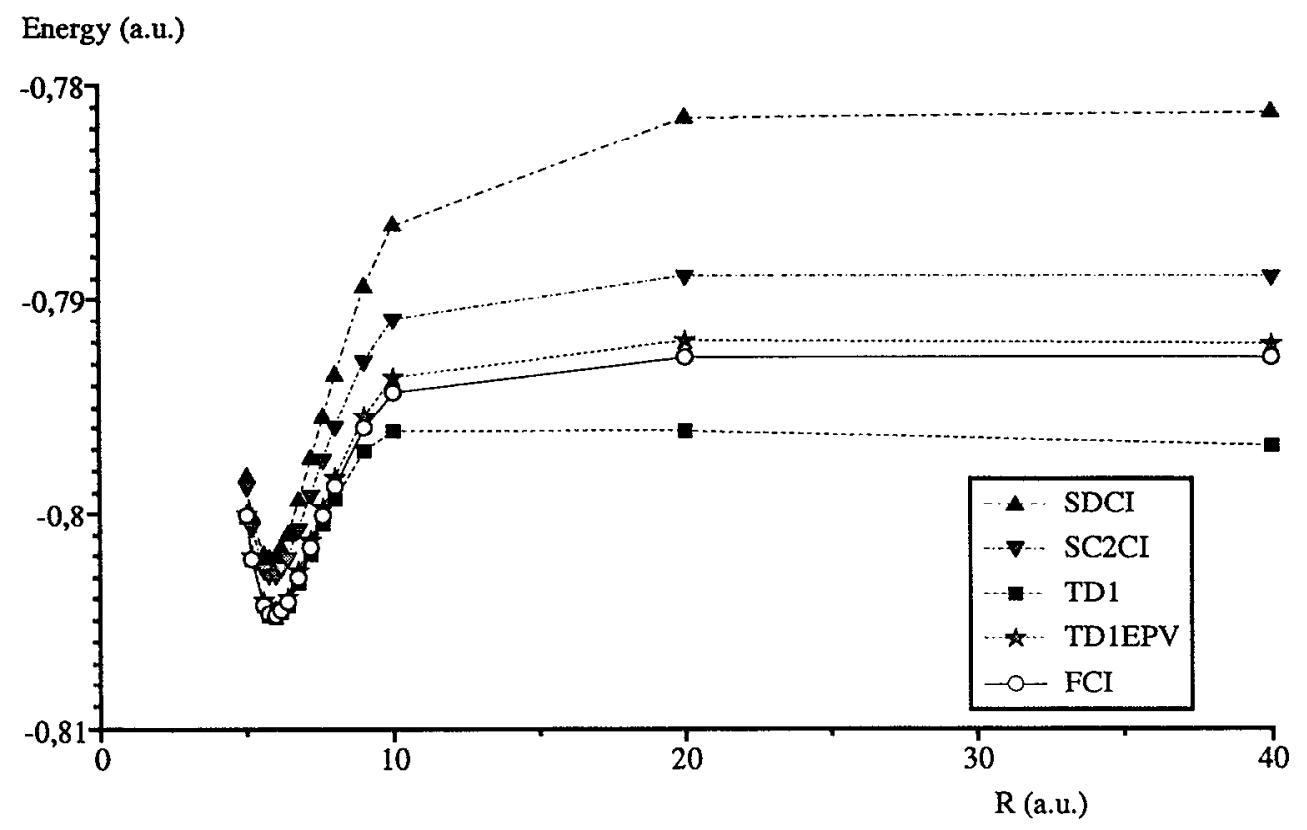

FIG. 2. Evolution of the energy in the T-shaped $\mathrm{Li}_{4}$ problem in a double zeta basis set, as a function of the length $R$ of the axial bond.

free, the method might equally be applied to excited states, at least when one may have a single reference zeroth-order description, as for most triplet states.

The dressing may take several forms. It may be concentrated on the first column, on the diagonal, or concern the full matrix, and the two first solutions have the advantage of economy of the information storage. The dressing may be expressed from the coefficients of the eigenvector on the outer space determinants, which have to be estimated. A further paper will proceed to a coupled-cluster type evaluation of these amplitudes, while the present work uses a perturbative evaluation, easily applicable to excited states.

We have essentially considered the case where the intermediate model space spanned the first order wave function (singles and doubles), and the useful outer space involved the space spanning the 2 nd order correction (triples and quadruples). Since the interaction between the outerspace determinants was not considered explicitely in our formulations, the method must be compared to the 4th order perturbative evaluation of the energy. The cost of our proposals should be similar to MP4 and the accuracy should be much better since the amplitude on the doubly excited state are variational, and actually our proposals do not diverge when a (near) degeneracy occurs in the model space.

The simplest perturbative scheme may be seen as a convenient reformulation of the so-called shifted- $B_{k}$ approximation or of the non-self-consistent 2 nd order intermediate Hamiltonians and therefore behaves correctly when the number of particles increases. In order to obtain this property, one must proceed to the diagonalization after dressing, instead of perturbing a multireference vector obtained by diagonalizing a bare matrix, as done in CIPSI (Ref. 22) and most of the related MRMP2 methods. ${ }^{23}$
The addition of EPV corrections greatly improves the behavior in case of strong degeneracies within the model space, at a low computational extra-cost when one stores the summation of the effect of double (and single) excitations in terms of one MO, two-MOs, three-MOs partial contributions, as previously proposed ${ }^{20}$ and used in the self-consistent size-consistent CI method. ${ }^{18}$ The numerical tests have shown the efficiency of the method.

The method is of single-reference character. However one must notice (i) that the choice of the reference is free. It may be an excited configuration as discussed above, and in case of avoided crossing, it may change, as shown on the $\mathrm{H}_{4}$ problem where one goes from $\phi_{0}=a^{2}, b^{2}$ to $\phi_{0}^{\prime}$ $=a_{1}^{2} b_{2}^{2}$. (ii) When the EPV contributions are considered, the method is able to treat the situations where one of the intermediate determinants has an amplitude equal to that of $\phi_{0}$.

That striking ability of the TD1-EPV algorithm to treat near degeneracies has been illustrated on $4 e^{-}$problems only, but it should remain valid for systems involving more electrons, since the amplitudes of the triples and quadruples are evaluated perturbatively in the intermediate normalization, avoiding size-consistency defects.

Of course one should consider the following generalizations: (i) The intermediate model space might be larger than the set of determinants interacting with $\phi_{0}$. As an extreme case it might also involve all the determinants belonging to the 2 nd order wave function $\psi^{(2)}$, and the method would then provide an improved evaluation of the sixth-order corrected energy. More reasonably, the intermediate model space might include a limited number of triples, quadruples, etc. If, for instance, one breaks a double bond, a valence quadruply excited configuration be- 
comes degenerate with $\phi_{0}$, and more generally the intermediate model space might include a CAS plus all the doubles. Of course the generation of the $\phi_{\alpha}$ 's has to be revised. (ii) On the contrary the intermediate space might be shorter than the whole set of all singles and doubles, containing only those of largest amplitudes. Then the contributing outer space will also concern the other singles and doubles, not all quadruples. The dressing will also concern the main model space determinant $\phi_{0}$,

$$
\left\langle\phi_{0}|\Delta| \phi_{0}\right\rangle=\sum_{i \notin S} C_{i}\left\langle\phi_{0}|H| \phi_{i}\right\rangle,
$$

the coefficients $C_{i}$ being evaluated to the first order, or preferably by the recently proposed nondivergent selfconsistent 2 nd order technique ${ }^{24}$ which proceeds by diagonalizing $2 \times 2$ matrices spanned by $\phi_{0}$ and $\phi_{i}$ after a minimal dressing by EPV. Then the vector on $S$ and $D$ is complete and the dressing of the $S$ and $D$ diagonal energies by the $T$ and $Q$ configurations may be performed according to the same technique.

In practice the implementation rests on the efficient algorithm recently proposed by Maynau and Heully for the perturbation of the SDCI vector by all triples and quadruples. ${ }^{15}$ But instead of perturbing this MR vector, one dresses the $S$ and $D$ configurations diagonal energies, in an iterative manner. The computation time per iteration is the same; a few iterations are necessary but while the SD(TQ) algorithm was not size-extensive, the present one is. A preliminary work by Maynau and two of $\mathrm{us}^{25}$ had given an approximate version of the present proposal; in that work the total-dressing energy was calculated as a mean-value of the diagonal dressing

$$
\epsilon=\langle\widetilde{\psi}|\Delta| \widetilde{\psi}\rangle
$$

on a vector resulting from the $(\mathrm{SC})^{2} \mathrm{SDCI}$ method. The $(\mathrm{SC})^{2} \mathrm{SDCI}$ method is size extensive and iterative, the vector $\widetilde{\psi}$ is certainly very close to the eigenvector of $P(H$ $+\Delta) P$. So that the iterations concerned an algorithm the cost of which is only that of SDCI, and not the long loops generating all triples and quadruples. But the present formulation is much clearer and certainly more reliable.

\section{ACKNOWLEDGMENTS}

The authors thank the Spanish CICYT (project PB 91-0634) and S.E.U.I. (project OP90-0042) as well as the C.C.E.C. of the Generalitat Valenciana for the financial support of this work and J. L. Heully for helpful remarks.

\footnotetext{
'J. Goldstone, Proc. R. Soc. London, Ser. A 239, 267 (1957).

${ }^{2}$ F. Coester, Nucl. Phys. 1, 421 (1958); F. Coester and H. Kummel, ibid. 17,477 (1960).
}

${ }^{3}$ B. H. Brandow, Rev. Mod. Phys. 39, 771 (1967).

${ }^{4}$ See, for instance, Ph. Durand and J. P. Malrieu, in Ab-initio Methods in Quantum Chemistry, edited by K. P. Lawley (Wiley, New York, 1986), Vol. 1, p. 321; D. Mukherjee and S. Pal, Adv. Quantum Chem. 20, 291 (1989); G. Hose and U. Kaldor, Chem. Phys. 62, 469 (1981); J. Phys. Chem. 86, 2133 (1982).

${ }^{5}$ B. Jeziorski and H. J. Monkhorst, Phys. Rev. A 24, 1668 (1981); L. Z Stolarczyk and H. J. Monkhorst, ibid. 32, 725, 743 (1985); W. D. Laidig and R. J. Bartlett, Chem. Phys. Lett. 104, 424 (1984); L. Meissner, S. A. Kucharski, and R. J. Bartlett, J. Chem. Phys. 91, 6187 (1989); B. Jeziorski and J. Paldus, ibid. 90, 2714 (1989); L. Meissner and R. J. Bartlett, ibid. 92, 561 (1990); A. Balková, S. A. Kucharski, L. Meissner, and R. J. Bartlett, ibid. 95, 4311 (1991); D. Mukhopadhyay and D. Mukherjee, Chem. Phys. Lett. 163, 171 (1989); 177, 441 (1991); V. Kaldor, Theor. Chim. Acta 80, 427 (1991); J. Paldus, P. Piecuch, L. Pylypow, and B. Jeziorski, Phys. Rev. A 47, 2738 (1993), and references therein.

${ }^{6}$ J. P. Malrieu, Ph. Durand, and J. P. Daudey, J. Phys. A 18, 809 (1985).

${ }^{7}$ A. V. Zaitserski and J. L. Heully, J. Phys. B 25, 603 (1992); see also, D. Mukherjee, Int. J. Quantum Chem. 20, 409 (1986); S. Koch, Theor. Chim. Acta 81, 169 (1991).

${ }^{8}$ S. Evangelisti, J. P. Daudey, and J. P. Malrieu, Phys. Rev. A 35, 4930 (1987).

${ }^{9}$ L. E. Nietsche and E. R. Davidson, J. Chem. Phys. 68, 3103 (1978); J. Am. Chem. Soc. 100, 7201 (1978); E. R. Davidson, L. B. McMurchie, and S. J. Day, J. Chem. Phys. 74, 5491 (1981).

${ }^{10}$ C. Bloch, Nucl. Phys. 6, 329 (1958).

${ }^{11}$ H. Feschbach, Ann. Phys. (NY) 5, 357 (1958); 19, 287 (1962); Ann. Rev. Nucl. Sci. 8, 49 (1958); P. O. Löwdin, J. Math. Phys. 3, 969 (1962).

${ }^{12}$ See, for instance, K. F. Freed, Acc. Chem. Res. 16, 137 (1983), and references herein; P. Westhaus, J. Chem. Phys. 73, 5197 (1980); S. Pal, M. D. Prasad, and D. Mukherjee, Theor. Chim. Acta 66, 311 (1984), and references herein; J. Y. Metz and J. Lievin, ibid. 67, 369, 391 (1985); B. Lam, M. W. Schmidt, and K. Ruedenberg, J. Phys. Chem. 89, 2221 (1985); F. Spiegelmann, J. P. Malrieu, D. Maynau, and J. P. Zurru, J. Chem. Phys. 83, 69 (1986); M. M. Goodgame and W. A. Goddard III, Phys. Rev. Lett. 54, 66 (1985); A. Clotet, J. P. Daudey, J. P. Malrieu, J. Rubio, and F. Spiegelmann, Chem. Phys. 147, 293 (1990).

${ }^{13}$ J. L. Heully and J. P. Malrieu, Chem. Phys. Lett. 199, 545 (1992).

${ }^{14} \mathrm{Z}$. Gershgorn and T. Shavitt, Int. J. Quantum Chem. 2, 751 (1968).

${ }^{15}$ D. Maynau and J. L. Heully, Chem. Phys. Lett. 211, 625 (1993).

${ }^{16}$ R. Cimiraglia, J. Chem. Phys. 83, 1746 (1985); R. Cimiraglia and M. Persico, J. Comput. Chem. 8, 39 (1987).

${ }^{17}$ J. P. Malrieu, Theor. Chim. Acta 62, 163 (1982).

${ }^{18}$ J. P. Daudey, J. L. Heully, and J. P. Malrieu, J. Chem. Phys. 99, 1240 (1993).

${ }^{19}$ H. P. Kelly and M. A. Sessler, Phys. Rev. 132, 2091 (1963); Phys. Rev. A 134, 1450 (1964); W. Meyer, Int. J. Quantum Chem. Symp. 5, 341 (1971); R. Alrichs, H. Lishka, V. Staemmler, and W. Kutzelnigg, J. Chem. Phys. 62, 1225 (1975).

${ }^{20}$ M. B. Lepetit and J. P. Malrieu, J. Chem. Phys. 87, 5937 (1987).

${ }^{21} \mathrm{~K}$. Jankovski and J. Paldus, Int. J. Quantum Chem. 18, 1243 (1980); K. Jankovski, J. Paldus, and J. Wasilewski, J. Chem. Phys. 95, 3549 (1991); K. Jankovski, J. Paldus, I. Grabowski, and K. Kowalski, ibid. 97, 1600 (1992).

${ }^{22}$ B. Huron, P. Rancurel, and J. P. Malrieu, J. Chem. Phys. 58, 5745 (1973); S. Evangelisti, J. P. Daudey, and J. P. Malrieu, Chem. Phys. 75, 91 (1983).

${ }^{23}$ R. J. Harrison, J. Chem. Phys. 94, 5021 (1991).

${ }^{24}$ M. B. Lepetit and J. P. Malrieu, Chem. Phys. Lett. 208, 503 (1993).

${ }^{25}$ J. Sanchez-Marin, D. Maynau, and J. P. Malrieu, Theor. Chim. Acta (in press). 